\title{
A PERFORMANCE AUDIT OF THE TRANSIENT ISCHAEMIC ATTACK SERVICE AT PENNINE ACUTE HOSPITALS NHS TRUST
}

Dr. Cheng Khuang LIM ${ }^{1}$, Geriatric Specialty Trainee; Dr. Cassandra NG², Geriatric Specialty Trainee; Dr. Rebecca OATES ${ }^{3}$, Geriatric Specialty Trainee and Dr. Aung SETT" Consultant Physician and

Stroke Medicine

${ }_{1}^{1}$ Salford Royal NHS Foundation Trust, ${ }^{2}$ Manchester University Hospitals NHS Foundation Trust, ${ }^{3}$ Bolton NHS Foundation Trust, 4 Pennine Acute Hospitals NHS Trust

\section{BACKGROUND}

Transient Ischaemic Attack (TIA) is increasingly diagnosed due to better public awareness. Approximately 46,000 people have their first TIA in the UK every year. Timely assessment and appropriate delivery of
treatment for TIA patients in the outpatient setting could significantly reduce developing stroke by $80 \%$. The National Clinical Guideline for Stroke recommends that all TIA suspects should be assessed and investigated at a specialist neurovascular clinic either within 24 hours for high-risk patients or within a maximum of 1 week for low-risk patients. The Sentinel Stroke National Audit Programme 2012 reported $75-87 \%$ o patients with TIA were seen within

However, there has been a rapid expansion and centralisation of the comprehensive Stroke Service at our Trust since 2012, there is increased demand of TIA referrals. Recent trust TIA assessment performance has shown that only just above possible high risk TIA were seen within 24 hours.

This may be due to a combination of factors, but as clinicians, inappropriate use of TIA referral forms has been identified in the clinics in neurovascular clinics.

\section{OBJECTIVES}

The aims of this audit were

To determine the efficient delivery of the neurovascular clinic service is in line with The National Clinical Guidelines for TIA assessment. To review the appropriateness of referrals sent to the neurovascular clinics.

To highlight possible areas for improvement especially in updating local TIA guideline and proforma for referrals to neurovascular clinics. To establish the effect on waiting times and appropriateness of
referrals following the introduction of a new referral proforma.

Our standards were:

1. All patients who have had a suspected TIA with ABCD2 score $\geq 4$ should have specialist assessment in neurovascular clinic within 24 hours. 2. All patients who have had a suspected TIA with ABCD2 score $<3$ should have specialist assessment in neurovascular clinic within 7 days. 3. Patients referred to the neurovascular clinic should have a history
consistent with a possible TIA, defined by a transient episode of neurologic dysfunction caused by focal brain, spinal cord, or retina ischemia with a sudden onset.

\section{METHODS}

The initial audit involved a retrospective review of all referrals to the neurovascular clinics across all four sites (North Manchester Genera Hospital, Fairfield General Hospital, Royal Oldham Hospital and Rochdale Royal Infirmary) from 1st July 2014 to 1st September 2014.

A list of patients received by the Central Bookings Office for the neurovascular clinic during the above 2 months period was generated, and a scanned copy of the referral form was accessed electronically on CDViews. Data was populated onto a Microsoft Excel sheet and analysed. This audit led to the introduction of a new TIA referral proforma to improve the quality of referrals to the neurovascular clinics.

In 2017, we conducted a re-audit using the same methodology. This looked at referrals from $20^{\text {th }}$ January 2017 to $20^{\text {th }}$ February 2017. Data was analysed using Microsoft Excel and the audit results were compared to baseline audit.

\section{RESULTS}

There were 325 patients in the $1^{\text {st }}$ audit and 128 patients in the $2^{\text {th }}$ audit, with an equal spread of males and females. Patients included in this audit aged between 25 years to 98 years.

Over half of the referrals were consistently received from General Practitioners and approximately 30\% from Accident and Emergency in both cycles of the audit. The remainder of referrals came from other specialties, such as Ophthalmology and Acute Medicine.

The initial audit highlighted less than satisfactory compliance with all three standards (see Table 1), with $27 \%$ of referrals received classified as not related to cerebrovascular disease (see Figure 1).

\begin{tabular}{|c|c|c|}
\hline Standards & $\begin{array}{l}\text { Compliance } \\
\text { (2014) }\end{array}$ & $\begin{array}{c}\text { Compliance } \\
\text { (2017) }\end{array}$ \\
\hline $\begin{array}{l}\text { 1. All patients who have had a } \\
\text { suspected TIA with } A B C D 2 \text { score } \geq 4 \\
\text { should have specialist assessment in } \\
\text { neurovascular clinic within } 24 \text { hours. }\end{array}$ & $57.4 \%$ & $56.1 \%$ \\
\hline $\begin{array}{l}\text { 2. All patients who have had a } \\
\text { suspected TIA with } A B C D 2 \text { score }<3 \\
\text { should have specialist assessment in } \\
\text { neurovascular clinic within } 7 \text { days. }\end{array}$ & $76.9 \%$ & $96.3 \%$ \\
\hline $\begin{array}{l}\text { 3. Patients referred to the } \\
\text { neurovascular clinic should have a } \\
\text { history consistent with a possible } \\
\text { TIA, defined by a transient episode } \\
\text { of neurologic dysfunction caused by } \\
\text { focal brain, spinal cord, or retinal } \\
\text { ischemia with a sudden onset. }\end{array}$ & $66.2 \%$ & $74.3 \%$ \\
\hline
\end{tabular}

Table 1. Percentage compliance with pre-defined standards

Following the introduction of a new referral proforma, the compliance with 2 out of 3 standards improved significantly (as shown in Table 1). The appropriateness of referrals increased from $66.2 \%$ to $74.3 \%$.

This change also saw the significant reduction in waiting times for clinic appointments. The mean waiting time for low risk TIAs decreased from 5.01 days to 3.82 days, but there was no significant change in the high risk TIAs cohort (see Figure 2).

The implementation of the new proforma was good, with $93 \%$ of referrals received in 2017 was using the new proforma as recommended.

Reasons for Referral (2014)

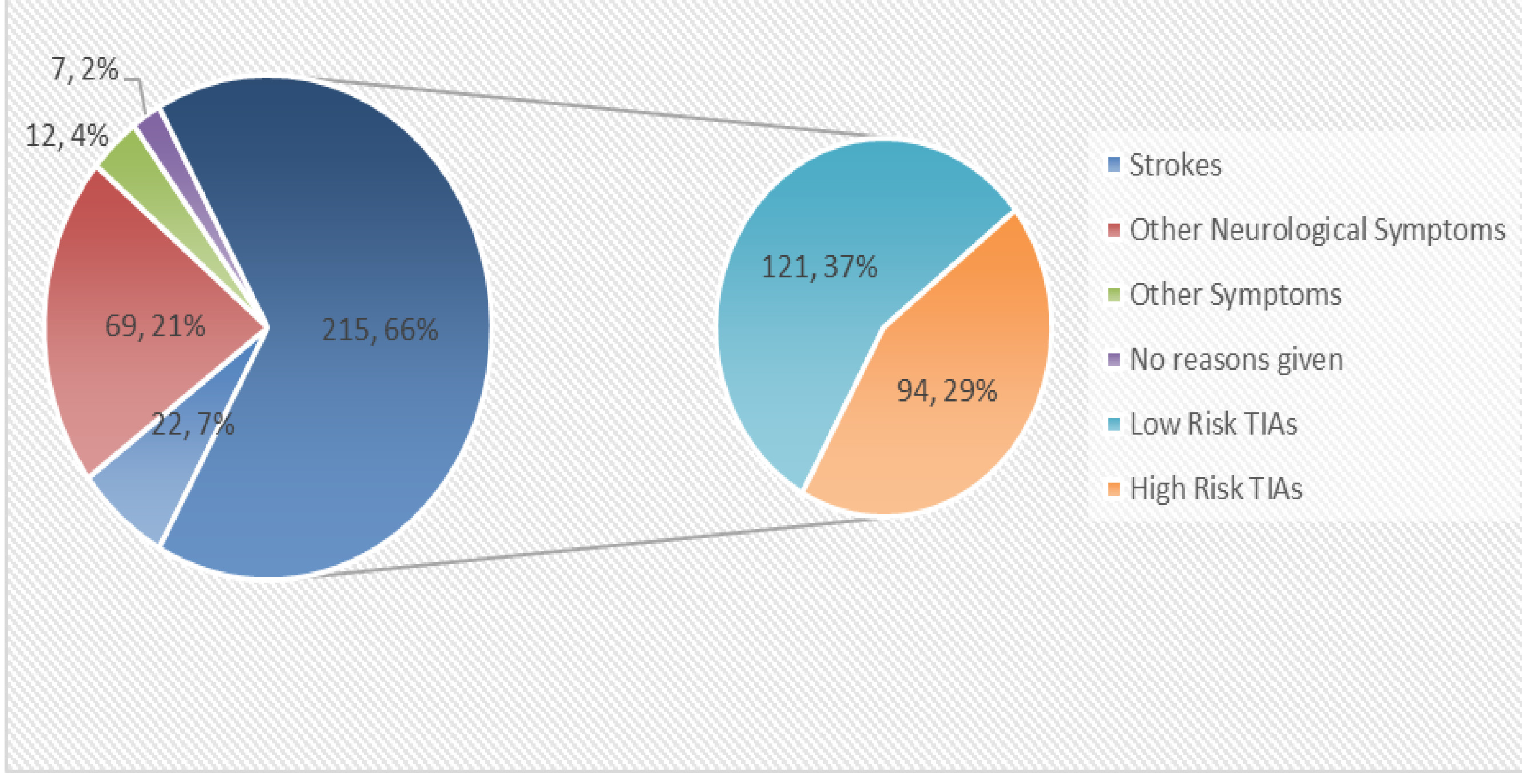

Reasons for Referral (2017)

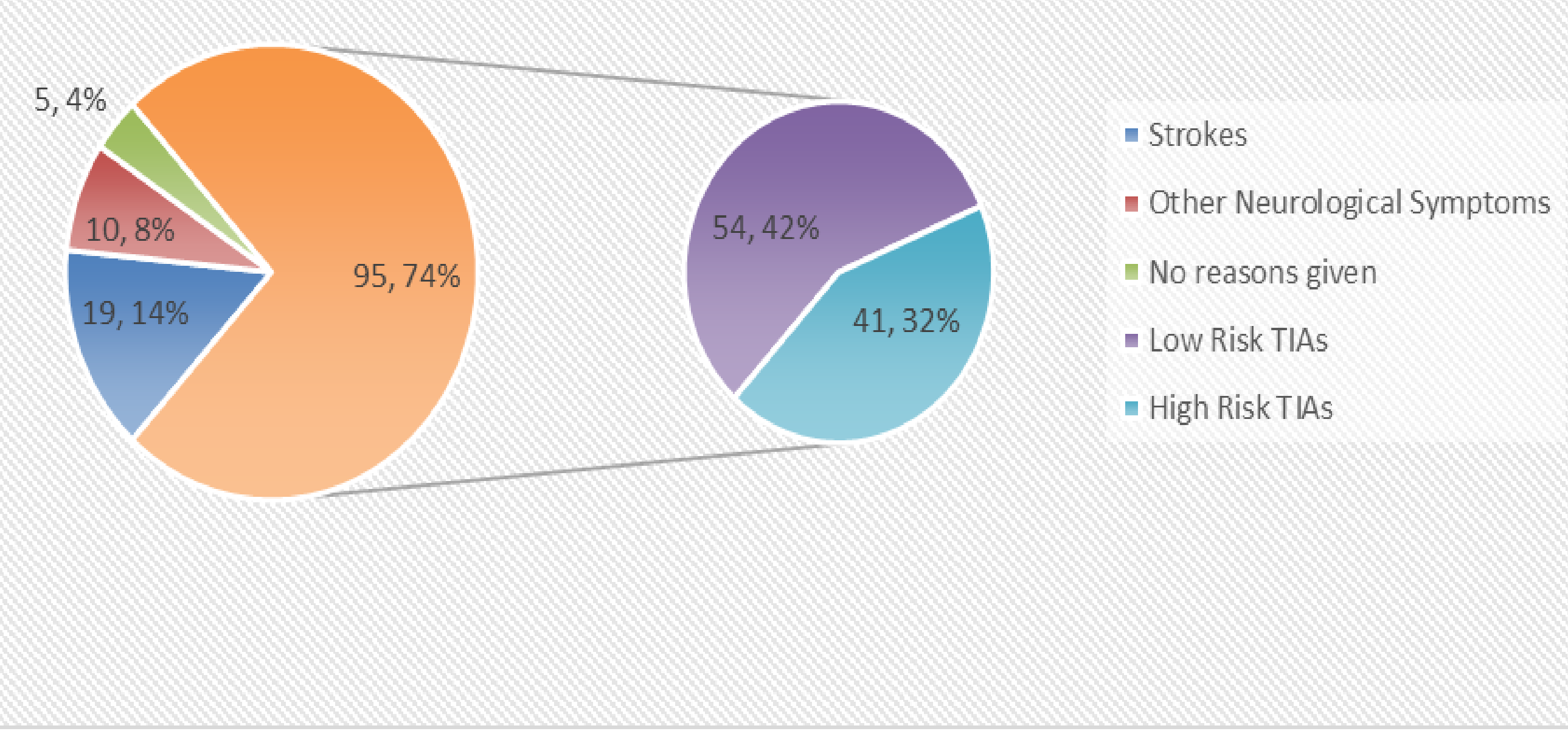

Figure 1. Reasons for referral to the Neurovascular Clinic in 2014 (above) and 2017 (below) Waiting Times for Appointments from Date of Receipt of
Referral

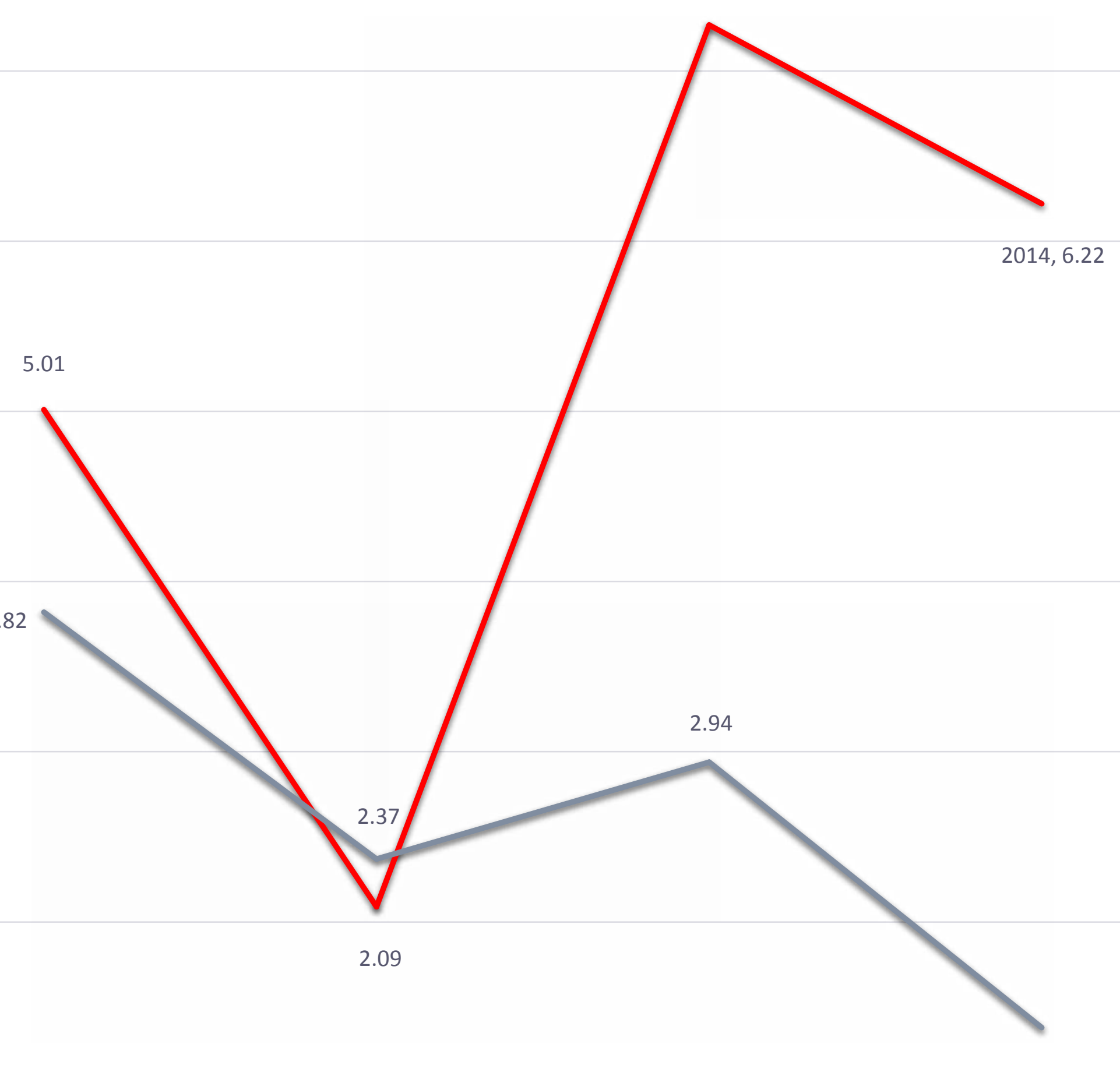

Figure 2. Average waiting times for appointments from date of receipt of referral

\section{DISCUSSION}

The initial audit has highlighted that the TIA service were not utilized appropriately. This has resulted in waste of resources and failure to provide timely service to patients who required specialist input, imaging and early treatment which could result in significant adverse outcomes.

In order to increase the number of patients seen within recommended timeframes, we have increased the numbers of neurovascular clinics, introduced new referral proforma and created information leaflets for patients. The introduction of the new proforma has improved the quality of the referrals and ensures more consistent and efficient service is provided to patients who have had a suspected TIA. This is supported by our study which showed that more than $90 \%$ of our patients were seen within 7 days. However, there were only $56.1 \%$ of high risk patients seen within 24 hours. Apart from expanding our service, we are looking into more convenient methods to contact patients with suspected TIA effectively. Instead of using traditional letter only, we are hoping to use SMS and emails to send appointment reminder to ensure good communication with patients.

In order to achieve sustainable improvement, we need to ensure continued use of online referral proforma at the front door. It is therefore important to ensure the delivery of education on the management of TIA at every induction course in Accident and Emergency departments. This helps to encourage the use referral proforma among juniors doctors who rotates regularly.

There were also limitations. We were unable to obtain referra requests for a number of patients on CDView for reasons that were unknown. Unfortunately, there was no alternative to look for the referral request and we had to exclude them in our study. There were also incomplete referral forms received. The information we received was inadequate and this had resulted in failure to safeguard the quality of referrals we received.

\section{CONCLUSIONS}

This concise and easy to use TIA proforma has improved the quality of referrals to the neurovascular clinics.

This may contribute to the increase in the number of patients seen in neurovascular clinics within recommended time frame.

We need to look into more effective ways to communicate with high risk patients to ensure that they are seen within 24 hours.

\section{CONFLICTS OF INTEREST}

